\title{
Anticipatory postural adjustments during self inflicted and predictable perturbations in Parkinson's disease
}

\author{
Mark L Latash, Alexander S Aruin, Ida Neyman, John J Nicholas
}

\begin{abstract}
This study investigated the relation between impaired anticipatory postural adjustments and bradykinesia in Parkinson's disease. Patients with Parkinson's disease and age matched controls stood on a platform. In one series of experiments, they performed fast, discrete shoulder flexion or extension movements. In another series, they were required to press a trigger with the right thumb and thus to release a load that was suspended from a bar which they were holding in front of them in extended arms. One more series included catching a load on the same bar. Anticipatory changes in the activity of postural muscles before fast voluntary movements occurred in patients and controls although the patients showed higher variability of anticipatory patterns. During load dropping and catching, control subjects had reproducible, although smaller, anticipatory changes in postural muscle activity. Such changes were absent in all but one patient. Two sources of these postural perturbations were analysed. The anticipatory postural adjustments in different muscle groups may counteract perturbations of different origin. The distal to proximal sequencing of joint involvement in postural reactions may be related to different reference points and working points associated with these tasks compared with reaching limb movements. The deficit in anticipatory postural adjustments in Parkinson's disease is likely to be unrelated to bradykinesia and is more likely to reflect the deficits in the basic processes of preparation and initiation of a motor act.
\end{abstract}

$(尹$ Neurol Neurosurg Psychiatry 1995;58:326-334)

Keywords: voluntary movement; Parkinson's disease; anticipation; electromyogram

When a standing person performs a fast voluntary arm movement, their postural equilibrium becomes endangered. Two factors contribute to such self inflicted postural perturbations. Firstly, the changes in limb and body geometry lead to a change in the projection of the centre of gravity. Secondly, the transmission of forces and torques from the moving limb through the body's linked segments leads to transient forces at other joints.
Thus movements, particularly fast ones, may be considered as postural perturbations. ${ }^{1}$ The CNS "expects" such perturbations and uses two types of corrective changes in the activity of postural muscles. ${ }^{2-8}$ Postural reactions of the first type are often termed anticipatory and are initiated by the subject's CNS in a feedforward manner ${ }^{4-12}$ based on predictions of expected postural perturbations. Later, after the movement initiation, compensatory reactions are triggered by sensory feedback signals and deal with actual perturbations of posture that occur due to suboptimal efficacy of the anticipatory reactions.

Postural deficits in Parkinson's disease ${ }^{13}$ involve changes in both groups of postural reactions. Firstly, there is a poorly controlled increase in the feedback triggered corrective reactions. ${ }^{14}$ Secondly, there is a deficit in the anticipatory changes in the activity of postural muscles. ${ }^{4}$ There is substantial variability among the studies of anticipatory postural adjustments in Parkinson's disease. These reports vary from minor changes in the anticipatory reactions, ${ }^{15}$ no differences in the timing of the early bursts seen on EMG in postural muscles but the amplitude of the bursts is decreased, ${ }^{16}$ smaller EMG changes in muscles involved in a postural component of a bimanual task that often lack anticipatory EMG changes, ${ }^{17}$ to the lack of anticipatory postural adjustments in $95 \%$ of patients with Parkinson's disease. ${ }^{18}$

Another prominent feature of Parkinson's disease is bradykinesia, ${ }^{13}$ - that is, slowness of voluntary movements that leads to smaller peak accelerations, and consequently, smaller forces perturbing the vertical posture. Such slow movements may not require an anticipatory corrective action. ${ }^{719}$ Thus bradykinesia may be the reason for the lack of anticipatory changes in the activity of postural muscles, because the CNS "knows" in advance that the perturbing forces will not endanger the equilibrium. So, some variability among the studies of anticipatory reactions in Parkinson's disease may reside in different motor tasks, different amounts of practice, and maybe other factors leading to different efficacy of the voluntary movements as postural perturbations.

To investigate the relation between bradykinesia and postural anticipation, we studied anticipatory postural adjustments in patients with Parkinson's disease and age matched controls during commonly used voluntary arm movements and also during dropping and catching loads from (into) extended hands. Postural perturbations induced by a 
self triggered load release or expected impact are predictable and independent of the ability of the subject to perform fast voluntary movements. Thus this procedure eliminates one source of ambiguity among the studies of postural adjustments in Parkinson's disease. Our preliminary findings in young control subjects showed that dropping and catching loads is associated with anticipatory postural adjustments seen, in particular, as changes in the background electrical activity of trunk, leg, and proximal arm muscles. ${ }^{2021}$

\section{Methods \\ SUBJECTS}

Six patients with idiopathic Parkinson's disease, (three women and three men, mean age $70 \cdot 1$ (SE 4.0)) were recruited from the inpatients treated in the Center for Rehabilitation at the Rush-Presbyterian St Luke's Medical Center. The duration of their symptoms ranged from one to 50 (mean 14.8 (SE 7.0)) years. The patients were referred by their primary physicians. According to the neurological assessment, two patients had stage III Parkinson's disease and four had stage III-IV Parkinson's disease. They had been admitted to hospital for an adjustment of their medications, and were tested after the adjustment was considered complete. All six patients were taking Sinemet combined with parlodel, eldepryl, and/or permax. Inclusion criteria comprised an ability to stand unassisted and to understand and follow the instructions involved in the testing procedure. Exclusion criteria comprised excessive tremor, cognitive disorders, hallucinations, history of other neurological disorders, and arthritis.

The control group consisted of six elderly subjects, (three women and three men, mean age $71 \cdot 2$ (SE 3.1)) without any known neurological disorders or arthritis.

All patients and controls gave informed consent according to the procedure approved by the human investigation committee of the Rush-Presbyterian St Luke's Medical Center.

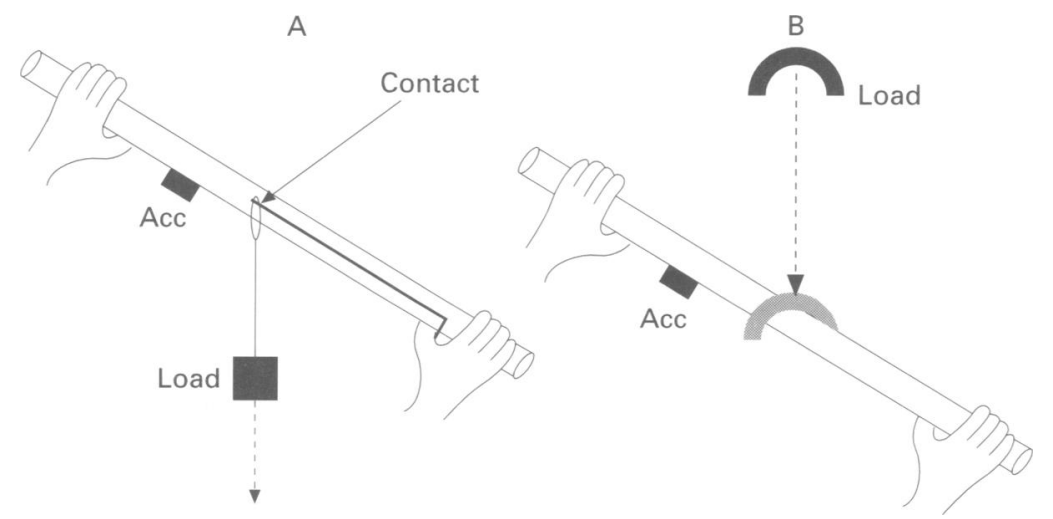

Figure 1 Schematic drawing of experiment 2. (A) The right thumb pressed the trigger releasing the load. Simultaneously, an electrical contact is broken. (B) The load is dropped from $0.5 \mathrm{~m}$ by the experimenter. The subject always sees the load. Acc = accelerometer.
APPARATUS

The subjects stood on a force platform (AMTI OR-6). The signals from the platform were amplified and used to measure reaction forces in three orthogonal directions (along the direction of gravity $\mathrm{Fz}$, parallel to the ground in the sagittal plane $\mathrm{Fx}$, and parallel to the ground in the frontal plane Fy) and moments of forces in two directions (in the sagittal (My) and frontal (Mx) planes). In the experiments with load manipulations (experiment 2), the subjects held a light aluminium bar with two foam rubber handles in front of them in both hands. In the experiments with unloadings (dropping a load), a $0.9 \mathrm{~kg}$ load was suspended on a short rigid metal cord from the centre of the bar. The bar was equipped with a trigger that required a light touch with the right thumb to release the load (fig 1A).

Three two axis goniometers (Penny and Giles) were taped on body segments and measured angles in the ankle (sagittal plane), knee, and hip (sagittal plane) joints. Acceleration was measured by a miniature unidirectional accelerometer (Sensotec). During the experiments with voluntary arm movements (experiment 1 ), the accelerometer was taped to the subject's right wrist and oriented so that its axis of sensitivity was in a sagittal plane. When dropping and catching loads (experiment 2), the accelerometer was taped to the aluminium bar that was used by the subject to release and catch the load. An electrical switch was used to signal the moment when the contact between the load and the bar broke (fig 1A).

Disposable paediatric electrocardiographic electrodes (Graphic Controls) were used to record the surface EMG activity of the anterior deltoid, posterior deltoid, rectus abdominis, erector spinae, rectus femoris, biceps femoris, soleus, and tibialis anterior muscles. The electrodes were placed over the muscle bellies. The distance between two electrodes of a pair was about $5 \mathrm{~cm}$. Signals from the electrodes were amplified $(\times 1600)$ and band-pass filtered $(60-500 \mathrm{~Hz})$. Later, these signals were rectified and filtered at $100 \mathrm{~Hz}$.

All the signals were sampled at $500 \mathrm{~Hz}$ with a 12 bit resolution. A Mac-IIci computer with customised software based on the LabView-II package was used to control the experiment, collect the data, and perform most of the analyses.

\section{PROCEDURE}

Experiment 1

Experiment 1 involved two series of movements. Each series involved six movements, and the subjects were asked to make movements within a series as similar to each other as they could. In the first series, the subjects were asked to perform bilateral shoulder extensions (backwards) "as fast as possible" over the nominal movement amplitude of $45^{\circ}$. In the second series, bilateral shoulder flexions (forward) "as fast as possible" over the nominal amplitude of $90^{\circ}$ were performed. Accuracy requirements were not stressed. 

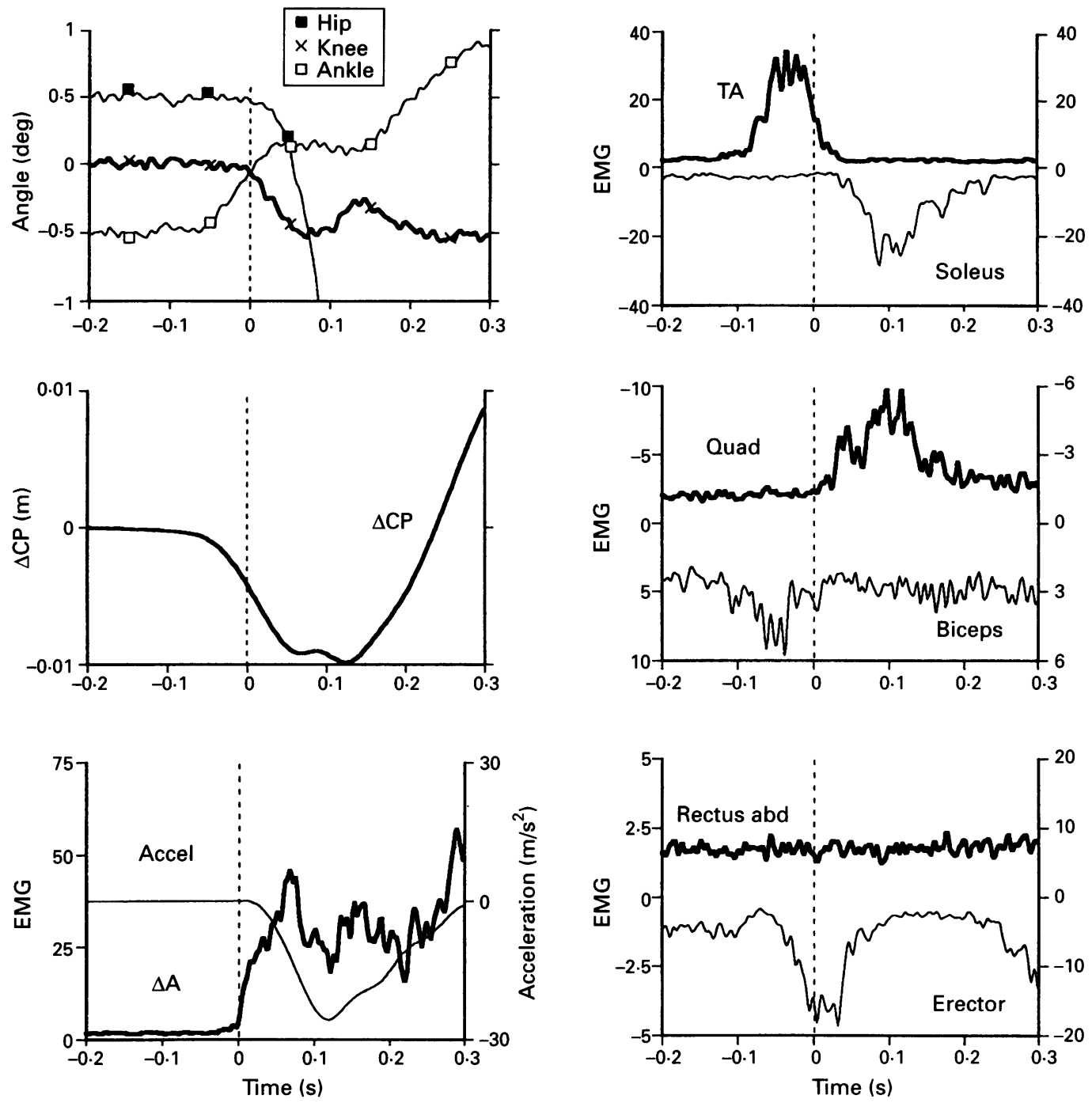

Figure 2 Kinematic and EMG patterns (averages of six trials) for a forward shoulder flexion movement performed "as fast as possible" by an elderly control subject. Before averaging, the trials were aligned according to the first visible change in the EMG of the anterior deltoid muscle $(\triangle A)$, which corresponds to time zero in the graphs. Note a distal to proximal order of joint involvement (left upper panel), an anticipatory backwards displacement of the centre of pressure ( $\triangle C P$, middle left panel), and an anticipatory activation of tibialis anterior, biceps femoris, and erector spinae muscles (right panels). EMGs of soleus, biceps femoris, and erector spinae muscles are inverted for better visualisation. Accel = acceleration of the right wrist; TA = tibialis anterior; Quad = rectus femoris, Rectus abd = rectus abdominis. EMG scales are in arbitrary units (bytes).

Before each series, the subjects were instructed which movements to perform and given three to five practice trials. Before each movement, the subjects were asked to stand quietly, their feet $0.3 \mathrm{~m}$ apart, their arms hanging loosely by their sides, with the palms towards the body. They were instructed, on hearing a tone signal (a beep), to perform the required movements with both arms "as fast as possible", to stop at the final position, to wait for a second beep, which came after a $3 \mathrm{~s}$ interval, to return back, and to wait for the next signal. The intervals between the trials within a series were $10 \mathrm{~s}$; the intervals between the series were about two minutes.

\section{Experiment 2}

In the first series of experiment 2, the subjects were instructed to stand quietly and to hold with two hands in front of them, in extended arms, the aluminium bar with the $0.9 \mathrm{~kg}$ load suspended from the bar on the short metal cord (fig 1A). At the tone signal, the subjects were required to press the trigger with the right thumb, thus releasing the load. The subjects were told that they had up to two seconds to perform the task and need not press the trigger as quickly as possible. The load was caught by the experimenter. Each subject was given a couple of practice trials to master the task of pressing the trigger. During the second series, the subjects were required to catch the same load on the same bar (fig 1B). The subjects stood in the same position holding the bar with both hands in front of them. The load (a $0.3 \mathrm{~m}$ long, $0.1 \mathrm{~m}$ wide belt filled with sand) was held by the experimenter 0.5 $\mathrm{m}$ above the centre of the bar. At the tone, the experimenter released the load, which hit the bar and hung on it. The subjects saw the load at all times.

\section{DATA PROCESSING}

The trials were viewed off line on a monitor screen and aligned according to the first visible change in a selected channel. This was 

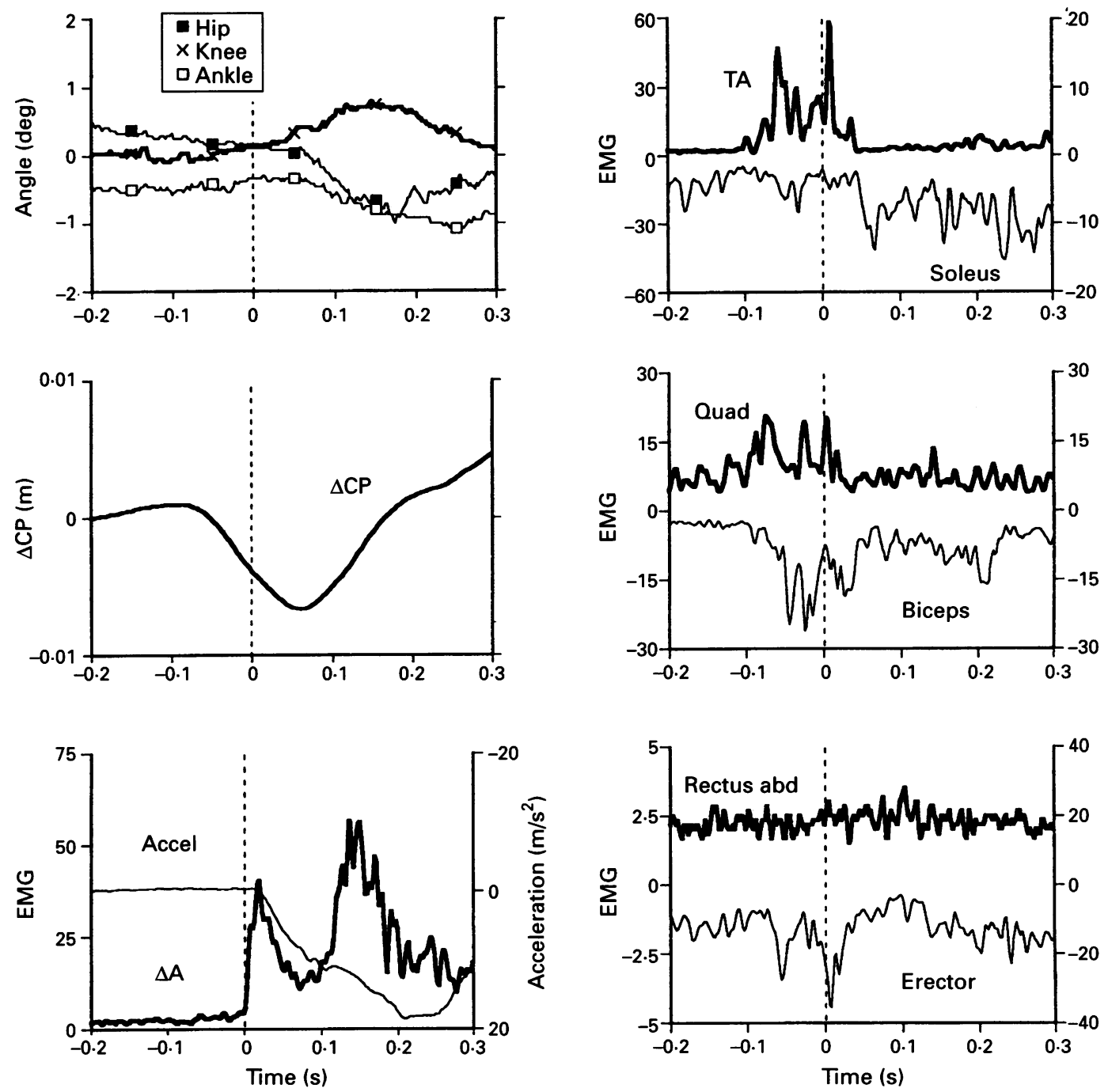

Figure 3 Kinematic and EMG patterns (averages of six trials) for a forward shoulder flexion movement performed "as fast as possible" by a patient with Parkinson's disease. Note an anticipatory backwards displacement of the centre of pressure (middle left panel), and an anticipatory activation of tibialis anterior, quadriceps, biceps femoris, and erector spinae muscles (right panels). The method of alignment and abbreviations are the same as in fig 2.

assigned time zero $\left(\mathrm{t}_{0}\right)$. Different methods of alignment were used: (1) In experiment 1, the trials were aligned by the first visible increase in the agonist EMG. That is, during shoulder extensions, the EMG signal from the posterior deltoid muscle was used, whereas during elbow flexion, the EMG signal from the anterior deltoid muscle was used. (2) In experiment 2, two types of data alignment were used. In the load catching trials, the first visible deflection of the acceleration signal was used for alignment. In the load dropping trials, the signal from the electrical switch was used. The signal from the accelerometer was used to double check the accuracy of the alignment. The agreement between the initial deviations of these two signals was virtually perfect in all the trials.

The following integral EMG measures were used for the leg and trunk muscles: (a) $\int 1$ : anticipatory activity-an integral from $-100 \mathrm{~ms}$ to $+50 \mathrm{~ms}$ with respect to $t_{0}$; (b) $\int 2$ : background activity-an integral from $-250 \mathrm{~ms}$ to $-100 \mathrm{~ms}$ with respect to $t_{0}$;

We are confident in referring to the EMG changes in the time interval from $-100 \mathrm{~ms}$ to $+50 \mathrm{~ms}$ as "anticipatory". In experiments with shoulder movements, our measurements of the time delays from $t_{0}$ to the first deviation of the signal from the accelerometer taped to the subject's wrist suggest that these delays are of the order of 25 to $30 \mathrm{~ms}$. If the minimal latency of the quickest, monosynaptic reflexes is added to this delay, the result is going to be over $50 \mathrm{~ms}$. So, our interval of integration did not allow any feedback based changes in the muscle activity. Also, in a pilot series, we compared EMG integrals over the period from $-100 \mathrm{~ms}$ to $+50 \mathrm{~ms}$ with the integrals from $-100 \mathrm{~ms}$ to $t_{0}$. There were no qualitative differences between these two measures.

The difference $\Delta \int=\left(\int 1-\int 2\right) / \int 2$ was used to characterise the anticipatory changes in the activity of the postural muscles. Horizontal displacements of the centre of pressure in the anterior-posterior $(\Delta \mathrm{CP})$ direction were calculated with the approximation: $\triangle \mathrm{CP}=$ $\mathrm{My} / \mathrm{Fz}$.

Statistical methods used variations of the Student's $t$ test and non-parametric statistics. 

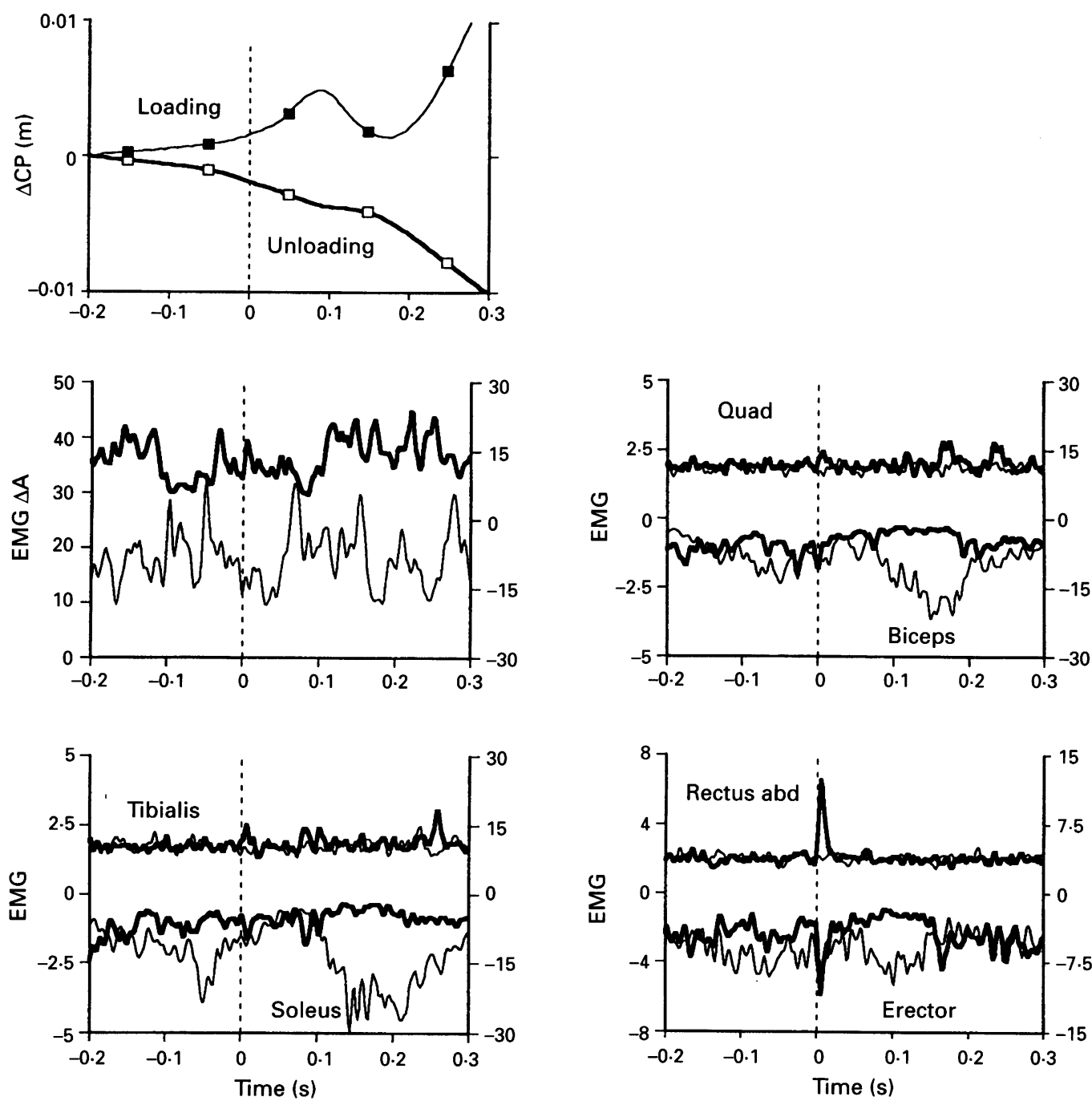

Figure 4 Kinematic and EMG patterns (averages of 6 trials) for a series of trials with dropping a $0.9 \mathrm{~kg}$ load (bold traces, unloading) and a series of trials involving catching a $0.9 \mathrm{~kg}$ load (thin traces, loading) performed by an elderly control subject. The method of alignment is explained in the methods section. Note an anticipatory backwards displacement of the centre of pressure during the unloading trials and an anticipatory forward displacement during the loading trials (the upper panel). During unloadings, anticipatory changes in the muscle activity include a small decrease in the activity of anterior deltoid muscle and simultaneous bursts of activity in rectus abdominis and erector spinae muscles. During loadings, a moderate increase in the activity of anterior deltoid, soleus, biceps femoris, and erector spinae was typically seen in control subjects. Abbreviations are the same as in fig 2.

\section{Results}

\section{EXPERIMENT}

Control subjects performed shoulder movements faster than patients with Parkinson's disease. Time to peak velocity (from $\mathrm{t}_{0}$ to the time when acceleration crossed zero for the first time) was about $50 \%$ higher in the patients. In particular, during shoulder flexions, average time to peak velocity was 263 (SD 25) $\mathrm{ms}$ for the control group and 390 (SD 58) $\mathrm{ms}$ for the patient group ( $P<0.005$, unpaired two tailed Student's $t$ test). Similarly, during elbow extensions, the average time to peak velocity was 208 (SD 25) ms for the control group and 328 (SD 58) $\mathrm{ms}$ for the patient group $(\mathrm{P}<0.001$, unpaired two tailed Student's $t$ test).

During arm movements, anticipatory reactions were seen in all control subjects and in five out of six patients with Parkinson's disease. Figure 2 illustrates typical patterns in a control subject during shoulder flexions.
There were apparent anticipatory bursts of activity seen on EMG in tibialis anterior, biceps femoris, and erector spinae muscles. This pattern of anticipatory changes in the background EMG activity was typical for the control subjects (see left upper panel, fig 6). Anticipatory postural adjustments were also seen in the backward displacement of the centre of pressure and in the changes in joint angles. The first visible joint displacement was in the ankle, followed by the knee, and later by the hip. During elbow extensions, the anticipatory bursts of activity were commonly seen in rectus abdominis, rectus femoris, and soleus muscles (see right upper panel, fig 6). They were accompanied by a forward displacement of the centre of pressure.

Figure 3 illustrates the kinematic and EMG patterns during elbow flexions in a patient with Parkinson's disease. The general pattern was similar to the one shown in fig 2 for a control subject. It included anticipatory EMG 

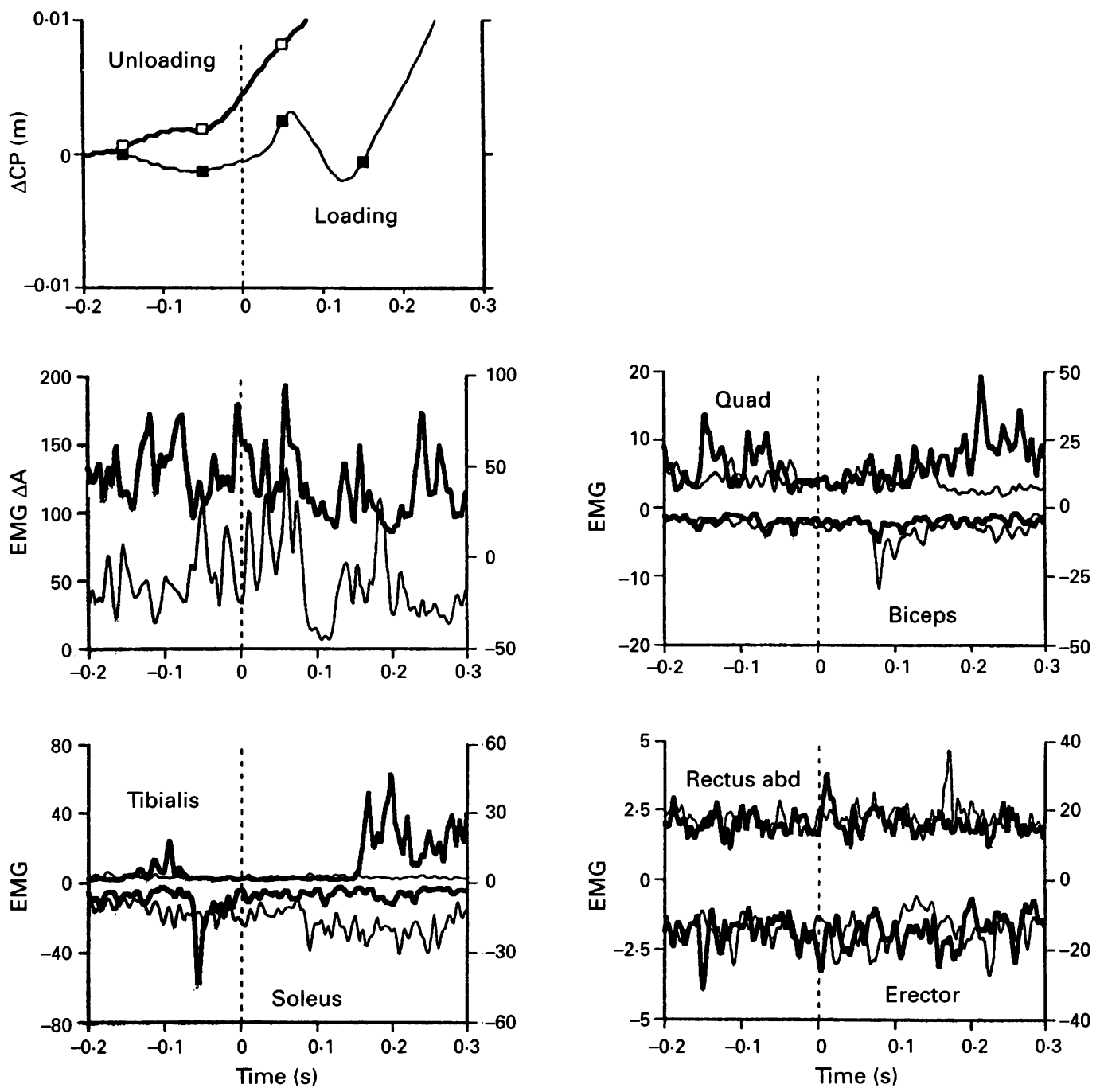

Figure 5 Kinematic and EMG patterns (averages of six trials) for a series of trials with dropping a $0.9 \mathrm{~kg}$ load (bold traces, unloading) and a series of trials involving catching a $0.9 \mathrm{~kg}$ load (thin traces, loading) performed by a patient with Parkinson's disease. This patient was the only one from our group who showed reproducible patterns of anticipatory postural adjustments. During unloadings, anticipatory changes in the muscle activity include an increase in the activity of tibialis anterior, soleus, and rectus femoris. During loadings, a moderate increase in the activity of anterior deltoid muscle can be seen. Abbreviations are the same as in fig 2.

bursts in tibialis anterior, biceps, and erector spinae muscles, and a backwards deviation of the centre of pressure. The only difference was in an early activation of quadriceps simultaneously with the EMG burst in biceps femoris. The leg joints showed smaller deviations without an obvious order of joint involvement. In general, patients with Parkinson's disease showed more variable patterns, sometimes with clear bursts of activity in only one or two of the leg muscles. During elbow extensions, the only obvious difference between the patients and control subjects was in an anticipatory activation of tibialis anterior in the patients and of the soleus in controls (see right upper panel, fig 6).

\section{EXPERIMENT 2}

During load catching and dropping, anticipatory reactions in postural muscles were less prominent in both groups of subjects as assessed by the $\Delta$ [EMG index (note the different scales in the upper and lower panels of fig 6). Despite being small, however, these differences reached statistical significance in a few cases.

Figure 4 illustrates averaged kinematic and EMG patterns in a control subject in the load dropping (bold traces) and catching (thin traces) series. Note simultaneous EMG bursts in erector spinae and rectus abdominis during the trials with load dropping (unloadings). Such bursts were typical for the control subjects although they did not occur in all the trials and, as a result, did not reach statistical significance when averaged across the subjects. Among other anticipatory events, there was a modest increase in the anterior deltoid, soleus, and biceps EMGs during the loading trials (catching the load) and a decrease in the anterior deltoid EMG in the unloading trials.

For the patient group, the only statistically significant anticipatory reaction was an increase in the activity of the anterior deltoid muscle in the loading trials. Only one patient with Parkinson's disease showed patterns of anticipatory adjustments in the leg and trunk muscles. Figure 5 shows his data. 
Shoulder flexion

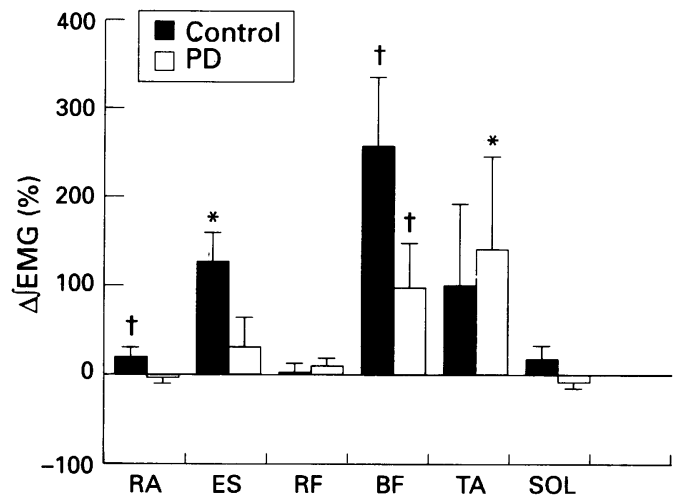

Loading

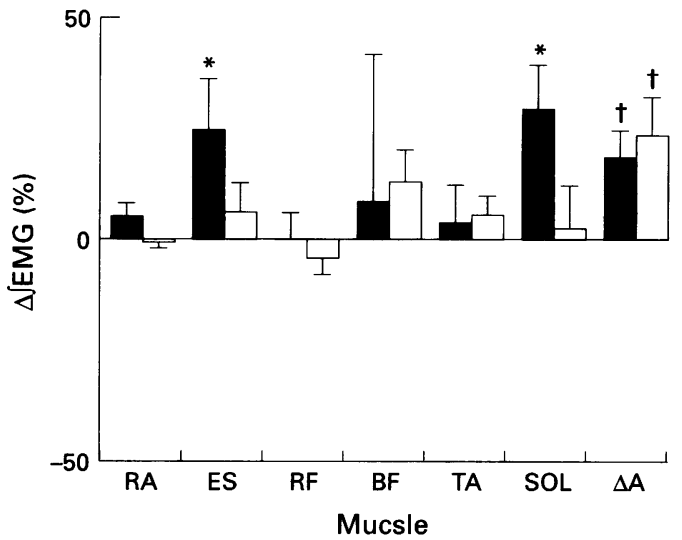

Shoulder extension

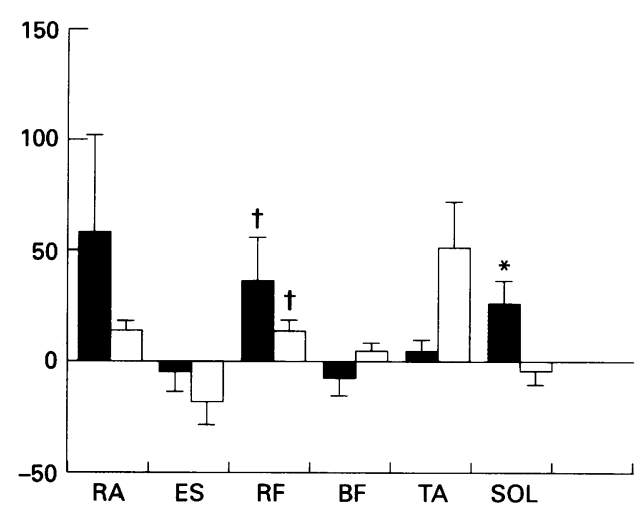

Unloading

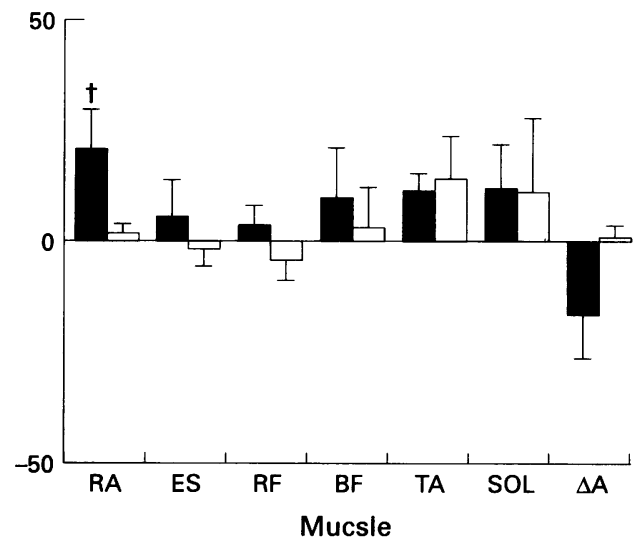

Figure 6 Mean values of the relative changes in background EMG activity ( $\triangle[E M G$ ) are shown with SE bars separately for control subjects (filled columns) and patients with Parkinson's disease (empty columns). Statistically significant changes in background EMG are shown by ${ }^{\star}(P<0.05$, single group, two tailed Student's t test $)$ or by $t(P<0 \cdot 05$, sign test $)$. $R A=$ rectus abdominis; $E S=$ erector spinae; $R F=$ rectus femoris; $B F=$ biceps femoris; $T A=$ tibialis anterior; $S O L=$ soleus; $\Delta A=$ anterior deltoid.

\section{Discussion}

ANTICIPATORY ADJUSTMENTS DURING FAST ARM MOVEMENTS

All six control subjects and five out of six patients with Parkinson's disease showed clear anticipatory changes in the activity of postural muscles during fast voluntary arm movements. These changes were seen in the patients despite the fact that their movements were considerably slower than the movements of the age and sex matched controls. These findings contrast with the reported lack of anticipatory postural adjustments in $95 \%$ of patients with Parkinson's disease ${ }^{18}$ and correspond better with the findings of relatively minor, quantitative changes in anticipatory postural reactions in such patients. ${ }^{151622}$ These reactions, however, were variable among the subjects, and particularly among the patients. This variability prevented some of the averaged indices from reaching statistical significance. Despite this, it is possible to identify a pattern of the anticipatory changes common for control subjects and a somewhat different pattern typical of the patients.

During forward arm movements (shoulder flexions), control subjects showed an increase in the background activity of proximal muscles at the dorsal part of the body (erector spinae and biceps femoris) and an increase in the activity of a distal muscle at the frontal part of the body (tibialis anterior). Earlier studies in control subjects more often described an increase in the activity of all the muscles at the dorsal portion of the legs and trunk, including the soleus, and a decrease in tibialis activity. ${ }^{2523}$ Backwards arm movements induced an increase in the activity of the proximal muscles at the frontal part of the body (rectus abdominis and quadriceps) and of a distal muscle at the dorsal part of the body (soleus). This pattern resembles our earlier findings in young control subjects. ${ }^{24}$

Patients with Parkinson's disease showed similar patterns of anticipatory postural adjustments with the only difference representing an increase in the activity of tibialis during shoulder movements in both directions. In an earlier study, we described a bimodal distribution of the changes in the activity of ankle flexors and extensors during shoulder movements in different directions. ${ }^{24}$ This distribution, as well as our findings of different patterns of anticipatory changes, imply the possibility of motor equivalencethat is, multiple strategies of muscle coordination during anticipatory postural adjustments to achieve a single motor outcome. ${ }^{25-27}$

There are two potential sources of postural perturbation during fast voluntary movements 
(see introduction). The first is transient and related to the reactive forces induced by a fast arm movement, whereas the second is related to the displacement of the projection of the centre of gravity. In our experiments, these two sources acted in different directions. During shoulder flexions, the reactive forces pushed the trunk backwards and were corrected by an anticipatory EMG burst in tibialis anterior. Activation of the biceps femoris and erector spinae counteracted the resulting forward displacement of the centre of gravity of the body. Similarly, during shoulder extensions, the reactive forces tried to move the trunk forward and were counteracted by an anticipatory EMG burst in the soleus. Activation of the quadriceps and rectus abdominis resisted the resulting backward displacement of the centre of gravity of the body.

In control subjects, we commonly saw a distal to proximal joint involvement in the anticipatory adjustments (fig 1). Similar findings have been reported for corrective postural reactions in children. ${ }^{28}$ The opposite, proximal to distal order of joint involvement is commonly seen in multijoint limb movements and is supposed to optimise the mechanical efficacy of muscle action. ${ }^{29}$ During limb movements, however, the "working point" is usually located at the distal end of the limb whereas the nearly motionless reference point is at one of the proximal joints-for example, at the shoulder during reaching with a hand. During standing, the motionless reference point is at the point of contact between the feet and the supporting surface whereas the "working point" may be associated with the position of the centre of gravity, which is closer to the hips-that is, to proximal joints. In this situation, a reversed sequencing of joint involvement becomes more efficient and is actually seen in the experiments. So, the apparent difference in the order of joint involvement during limb movements and during postural adjustments disappears if one takes into account that the terms "distal" and "proximal" make sense only when referred to a reference point that may be located somewhere on the trunk or, alternatively, on the tip of a limb.

\section{ANTICIPATORY ADJUSTMENTS DURING \\ LOADINGS AND UNLOADINGS}

Originally, we expected the alleged differences between the anticipatory reactions in patients with Parkinson's disease and elderly control subjects to be eliminated or attenuated during self inflicted or predictable perturbations that are independent of the subject's ability to make fast movements - that is, during load catching and dropping. The results did not fit our expectations. In general, control subjects showed comparably reproducible patterns of anticipatory postural adjustments during both experiment 1 and experiment 2 although the absolute values on the anticipatory changes in EMG were smaller during load dropping and catching (experiment 2). In experiment 2, however, five out of six patients with Parkinson's disease had poorly pronounced, variable patterns of anticipatory adjustments with the only exception of an increase in the activity of the anterior deltoid muscle in the load catching trials (see later). Only one patient, whose data are illustrated in fig 5, showed consistent anticipatory changes in the activity of the trunk and leg muscles during both load dropping and catching.

So, we are forced to conclude that the differences in anticipatory programming in Parkinson's disease are unrelated to bradykinesia and have a deeper origin possibly related to the basic processes of preparation and initiation of a motor action. These processes have been shown to deteriorate in Parkinson's disease. ${ }^{26}{ }^{30-32}$ We think, however, that our present knowledge is insufficient to allow us to formulate a viable hypothesis of which particular aspects of decision making, preparation of a motor programme, and its initiation give rise to the differences in postural anticipation found.

In load experiments, both sources of postural perturbation, the one related to transient forces at the load impact (release) and the one related to the displacement of the centre of gravity, acted in the same direction. That is, dropping (catching) the load induced a transient perturbation backwards (forward) and a shift of the centre of gravity in the same direction. As a result, anticipatory reactions in postural muscles in control subjects displayed a pattern often including simultaneous bursts in trunk muscles (fig 4) as well as simultaneous action of all the muscles of the dorsal part of the body including the proximal erector spinae and the distal soleus. A similar pattern of coactivation of trunk muscles has recently been described by Cresswell et al. ${ }^{33}$

Although the load in our experiments was rather small $(0.9 \mathrm{~kg})$, holding it in extended arms required a considerable level of activation of the anterior deltoid muscle. This muscle also showed anticipatory changes in its activity decreasing it when unloading was expected (load dropping) and increasing it before the load impact in the catching trials. Patients with Parkinson's disease showed an increase in the activity of the anterior deltoid muscle in the catching trials but no anticipatory changes in its activity during load dropping. This was the only reproducible sign of anticipatory pre-programming in our patients during experiment 2 .

\section{CAN AN ANTICIPATORY REACTION BE} TRIGGERED BY A TRIGGER?

Recently, some studies have suggested that it is not sufficient to have a predictable postural perturbation for the CNS to be able to generate anticipatory postural adjustments. ${ }^{34-36}$ In particular, if a perturbation coincides in time with a metronome or even if it is triggered by the subject by pressing a button, no anticipatory postural adjustments occur. To get these reactions, a major action by a large muscle group, including proximal muscles, is required.

In our experiment 2, load release was done by the subjects with a minimal action 
(pressing a trigger with the right thumb), while load impact was predictable but in no way controlled by the subject. Both manipulations induced anticipatory reactions in proximal arm, trunk, and leg muscles in our elderly control subjects. ${ }^{33}$ Thus we conclude that, in our particular context, predictability of a postural perturbation by itself was sufficient for the CNS to generate anticipatory postural adjustments. Our data are similar to anticipatory adjustments of arm position before load impact $^{21}$ and postural adjustments before landing after a jump. ${ }^{37-39}$

\section{CONCLUDING COMMENTS}

Before this study, we expected to see in patients with Parkinson's disease clear anticipatory changes in the activity of postural muscles during load perturbations but not during voluntary arm movements. Such data would have allowed us to make a direct link between bradykinesia and impaired anticipatory adjustments. The actual results were, however, different. We found only minor changes in the anticipatory patterns during voluntary arm movements in the patients compared with age matched controls, and no anticipatory adjustments in the leg and trunk muscles during load catching and dropping in five out of six patients. Apparently, we should speak not about impaired anticipation but rather about changed anticipation in Parkinson's disease, which is likely to reflect the primary pathological changes within the CNS and result in impaired processes of preparation and initiation of voluntary movements.

We are most grateful to the patients and control subjects for their exceptional patience and cooperation. This study was in part supported by a grant HD30128 from the National Center for Medical Rehabilitation Research, NIH, and by a grant from the Rush University Committee on Research.

1 Bernstein NA. The co-ordination and regulation of move ments. Oxford: Pergamon Press, 1967.

2 Cordo PJ, Nashner LM. Properties of postural adjustments associated with rapid arm movements. I Neurophysiol 1982;47:287-302

3 Bouisset S, Zattara M. Anticipatory postural movements related to a voluntary movement. In: Physiologie Spatiale. Toulouse: Cepadues Editions, 1983:137-41

4 Bouisset S, Zattara M. Segmental movement as a perturbation to balance? Facts and concepts. In: Winters JM Woo SL-Y, eds. Multiple muscle systems. Biomechanics and movement organization. New York: Springer-Verlag, 1990:498-506.

5 Friedli WG, Hallett M, Simon SR. Postural adjustments associated with rapid voluntary arm movements. I Electromyographic data. $\mathcal{F}$ Neurol Neurosurg Psychiatry 1984;47:611-22

6 Brown JE, Frank FS. Influence of event anticipation of postural actions accompanying voluntary movement. Exp Brain Res 1987;67:645-50.

7 Crenna P, Frigo C, Massion J, Pedotti A. Forward and backward axial synergies in man. Exp Brain Res 1987 65:538-48.

8 Massion J. Movement, posture and equilibrium: Interaction and coordination. Prog Neurobiol 1992;38: $35-56$

9 Hugon M, Massion J, Wiesendanger M. Anticipatory postural changes induced by active unloading and comparison with passive unloading in man. Pflugers Arch 1982 393:292-6.

10 Burbaud P, Garanx F, Gross CH, Bioulac B. Postura adjustments in the monkey: Effects of velocity on EMG sequence. Neurosci Lett 1988;84:51-6.
11 Ramos CF, Stark LW. Postural maintenance during movement: Simulations of a two joint model. Biol Cyber 1990;63:363-75.

12 Rogers MW, Pai Y-C. Dynamic transitions in stance support accompanying leg flexion movements in man. Exp Brain Res 1990;81:398-402.

13 Fahn S. Akinesia. In: Berardelli A, Benecke R, Manfredi M, Marsden CD, eds. Motor disturbances II. London: Academic Press, 1990:142-50.

14 Schieppati M, Nardone A. Free and supported stance in Parkinson's disease. The effect of posture and "postura set" on leg muscle responses to perturbation, and its relation to the severity of the disease. Brain 1991;114: 1227-44.

15 Diener HC, Dichgans J, Guschlbauer B, Bacher $M$, Langenbach $P$. Disturbances of motor preparation in basal ganglia and cerebellar disorders. Prog Brain Res 1989;80:481-8.

16 Dick JPR, Rothwell JC, Berardelli A, Thompson PD Gioux M, Benecke R, Day BL, Marsden CD. Associated postural adjustments in Parkinson's disease. Associated postural adjustments in Parkinson's
f Neurol Neurosurg Psychiatry 1986;49:1378-85.

17 Viallet F, Massion J, Massarino R, Khalil R. Performance of a bimanual load-lifting task by Parkinsonian patients. $f$ Neurol Neurosurg Psychiatry 1987;50:1274-83.

18 Bazalgette D, Zattara M, Bathien N, Bouisset S, Rondo $P$. Postural adjustments associated with rapid voluntary arm movements in patients with Parkinson's disease. Adv Neurol 1986;45:371-4.

19 Horak FB, Esselman PE, Anderson ME, Lynch MK. The effects of movement velocity, mass displaced and task certainty on associated postural adjustments made by normal and hemiplegic individuals. $尹$ Neurol Neurosurg Psychiatry 1984;47:1020-8.

20 Aruin AS, Nicholas JJ, Gottlieb GL, Lee KC, Latash MI Anticipatory reactions during dropping and catching Anticipatory reactions during dropping and
weights. Fournal of Biomechanics 1994;27:604.

21 Lacquaniti F, Maioli C. The role of preparation in tuning anticipatory and reflex responses during catching. f Neurosci 1989;9:149-59.

22 Latash L, Aruin AS, Neyman I, Nicholas JJ, Shapiro MB Feedforward postural reactions in patients with Parkinson's disease in a two-joint motor task Electroencephalogr Clin Neurophysiol 1995 (in press).

23 Bouisset S, Zattara $M$. Biomechanical study of the programming of anticipatory postural adjustments associated with voluntary movement. fournal of Biomechanics 1987;20:735-42.

24 Aruin AS, Latash ML. Directional specificity of postural muscles in feedforward postural reactions during fast voluntary arm movements. Exp Brain Res 1995 (in press).

25 Berkinblit MB, Feldman AG, Fukson OI. Adaptability of innate motor patterns and motor control mechanisms. innate motor patterns and motor

26 Latash ML. Control of human movement. Champaign Human kinetics, 1993.

27 Keshner EA. Vertebral orientation and muscle activation patterns during controlled head movements in cats. Exp Brain Res 1994;98:546-50.

28 Shumway-Cook A, Woollacott MH. Dynamics of postural control in the child with Down syndrome. Physical Therapy 1985;65:1315-22.

29 van Ingen Schenau GJ. From rotation to translation: constraints on multi-joint movements and the unique action of biarticular muscles. Human Movement Science 1989;8: of biarticulit.

30 Evarts EV, Teravainen H, Calne DB. Reaction time in Parkinson's disease. Brain 1981;104:167-86.

31 Stelmach GE, Worringham CJ, Strand EA. Movement preparation in Parkinson's disease: the use of advance information. Brain 1986;109:1179-94.

32 Brown RG, Marsden CD. Dual task performance and processing resources in normal subjects and patients with Parkinson's disease. Brain 1991;114:215-31.

33 Cresswell AG, Oddsson L, Thorstensson A. The influence of sudden perturbations on trunk muscle activity and intra-abdominal pressure while standing. Exp Brain Res 1994;98:336-41.

34 Dufosse M, Hugon M, Massion J. Postural forearm changes induced by predictable in time or voluntary triggered unloding in man. Exp Brain Re 1985;60:330-34.

35 Paulignan Y, Dufosse M, Hugon M, Massion J. Acquisition of coordination between posture and movement in a tion of coordination between posture and movem

36 Struppler A, Gerilovsky L, Jakob C. Self-generated rapid taps directed to the opposite forearm in man: anticipatory reduction in the muscle activity of the target arm. Neurosci Lett 1993;159:115-8.

37 Melvill Jones G, Watt DGD. Observations on the control of stepping and hopping movements in man. $f$ Physiol

38 McKinley PA, Smith JL. Visual and vestibular contributions to prelanding EMG during jump-down in cats. Exp Brain Res 1983;52:439-48.

39 Dyhre-Poulsen P, Laursen AM. Programmed electromyographic activity and negative incremental muscle stiffness in monkeys jumping downward. $\mathcal{f}$ Physiol 1984 350:121-36. 